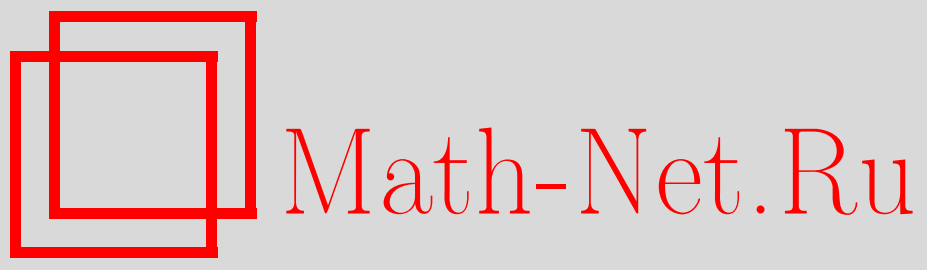

Б. М. Гуревич, А. А. Темпельман, Хаусдорфова размерность множества типичных точек для гиббсовских мер, Функи. анализ и его прил., 2002, том 36, выпуск 3, 68-71

DOI: https://doi.org/10.4213/faa207

Использование Общероссийского математического портала Math-Net.Ru подразумевает, что вы прочитали и согласны с пользовательским соглашением

http://www . mathnet.ru/rus/agreement

Параметры загрузки:

IP : 54.198 .64 .247

26 апреля 2023 г., $11: 46: 26$

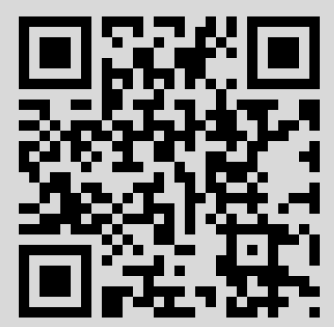




\title{
ЛиТЕРАТУРА
}

1. Арнольд В. И. Особенности каустик и волновых фронтов. Библиотека математика, т. 1, Фазис, М., 1996. 2. Giblin P. J., Holtom P. A. In: Geometry and Topology of Caustics, Banach Center Publications, Vol. 50 (S. Janeczko and V. M. Zakalyukin, eds.), Warsaw, 1999, pp. 91-105. 3. Goryunov V. V. In: Theory of Singularities and its Applications (V. I. Arnold, ed.), Adv. Soviet Math., Vol. 1, Amer. Math. Soc., Providence, 1990, pp. 157-200. 4. Holton P. Local central symmetry for Euclidean plane curves, Math. Sci. Dissertation, University of Liverpool, Sept. 1997. 5. Su Bu-chin, Affine differential geometry. Science Press, Beijing, Gordon and Breach, New-York, 1983. 6. Janeczko S. Geom. Dedicata, 60 (1996), 9-16. 7. Закалюкин В. М. Труды МИРАН, 209, 133-142 (1995). 8 Giblin P. J., Zakalyukin V. M. Singularities of centre symmetry sets. Preprint. Dept. of Math. Univ. of Liverpool, 2001.

Университет Ливерпуля, Великобритания email: pfgiblin@liverpool.uk

Московский государственный университет

email: vladimir@zakal.mccme.ru
Поступило в редакцию 25 декабря 2001 г.

УДК 517.938

\section{Хаусдорфова размерность множества типичных точек для гиббсовских мер*}

\author{
(C) 2002. Б. М. ГуревИЧ, А. А. ТЕмПЕЛЬМАН
}

Введем на множестве $X=V^{\mathbb{Z}^{d}}=\left\{x: \mathbb{Z}^{d} \rightarrow V\right\}$, где $d \geqslant 1,|V|<\infty(|V|-$ мощность множества $V$ ), топологию прямого произведения, отвечающую дискретной топологии на $V$, и определим группу преобразований $\tau=\left\{\tau_{s}, s \in \mathbb{Z}^{d}\right\}$ пространства $X$ равенством $\left(\tau_{s} x\right)(t)=x(s+t), x \in X, t \in \mathbb{Z}^{d}$. Пусть $\mathscr{P}-$ множество всех вероятностных борелевских мер на $X$ и $\mathscr{I}-$ совокупность $\tau$-инвариантных мер из $\mathscr{P}$. На пространстве $\mathscr{C}(X)$ непрерывных функций $f: X \rightarrow$ $\mathbb{R}$ определим функционал (давление)

$$
f \mapsto P(f):=\sup _{\nu \in \mathscr{I}}[h(\nu)-\nu(f)]
$$

где $h(\nu)$ - энтропия меры $\nu$ и $\nu(f)=\int f d \nu$. Мера $\mu \in \mathscr{I}$ называется равновесной относительно $f$ (или $f$-равновесной), если $h(\mu)-\mu(f)=P(f)$ (подробности см. в $[1,2])$. Множество $f$-равновесных мер обозначим через $\mathscr{E}(f)$. Назовем функцию $f \in \mathscr{C}(X)$ регулярной, если существует такая последовательность конечных множеств $F_{n} \subset \mathbb{Z}^{d}$, что $F_{n} \uparrow \mathbb{Z}^{d}$ при $n \rightarrow \infty$ и

$$
\lim _{n \rightarrow \infty}\left|F_{n}\right| \max _{x, y:\left.x\right|_{F_{n}}=\left.y\right|_{F_{n}}}|f(x)-f(y)|=0 .
$$

Если $f$ - регулярная функция, то все $f$-равновесные меры являются гиббсовскими мерами с одним и тем же потенциалом, имеющим конечную норму.

* Работа первого автора частично поддержана РФФИ, грант 02-01-00444. 
Рассмотрим последовательность кубов $T_{n}=[-n, n]^{d} \cap \mathbb{Z}^{d}$. Положим $C_{n}(x)=$ $\left\{y \in X:\left.x\right|_{T_{n}}=\left.y\right|_{T_{n}}\right\}$ и для $f \in \mathscr{C}(X), \alpha \in \mathbb{R}$ определим множество

$$
X_{f, \alpha}=\left\{x \in X: \lim _{n \rightarrow \infty} \frac{1}{\left|T_{n}\right|} \sum_{t \in T_{n}} f\left(\tau_{t} x\right)=\alpha\right\} .
$$

Назовем точку $x \in X$ типичной для меры $\mu \in \mathscr{I}$ (или $\mu$-типичной), если $x \in X_{f, \mu(f)}$ при всех $f \in \mathscr{C}(X)$. Множество $\mu$-типичных точек обозначим через $X(\mu)$. Согласно эргодической теореме Винера, $\mu\left(X_{f, \mu(f)}\right)=1$, если $\mu-$ эргодическая (относительно $\tau$ ) мера. Из сепарабельности пространства $\mathscr{C}(X)$ вытекает, что в этом случае также $\mu(X(\mu))=1$. Если же $\mu-$ неэргодическая мера, то $\mu(X(\mu))=0$.

Нас будет интересовать хаусдорфова размерность $\operatorname{dim}_{\rho}$ множеств $X_{f, \alpha}$ и $X(\mu)$ по отношению к метрикам $\rho$, которые определяются следующим образом. Пусть $\mathbf{a}=\left\{a_{n}\right\}-$ последовательность положительных функций $a_{n}: X \rightarrow \mathbb{R}$ со следующими свойствами:

(a) $a_{n}(x)=a_{n}(y)$, если $C_{n}(x)=C_{n}(y)$;

(b) $a_{n}(x) \downarrow 0$ при $n \rightarrow \infty$ и любом $x \in X$;

(c) существует такое $\gamma_{\mathbf{a}} \in \mathbb{R}$, что $\lim _{n \rightarrow \infty}\left|T_{n}\right|^{-1} \ln a_{n}(x)=\gamma_{\mathbf{a}}$ при всех $x \in$ $\bigcup_{\mu \in \mathscr{I}} X(\mu)$

(условие (c), очевидно, выполняется, если последний предел равен $\gamma_{\mathbf{a}}$ при всех $x \in X)$. Метрика $\rho$ называется согласованной с последовательностью а (ср. $[3,4])$, если $\operatorname{diam}_{\rho}\left(C_{n}(x)\right) \leqslant a_{n}(x)$ при всех $n \geqslant 0, x \in X$ и найдется такое $b>0$, что $\rho(x, y) \geqslant b a_{n}(x)$ при всех $x \in X, y \notin C_{n}(x)$ (это условие можно ослабить, предположив конечность индекса Морана, см. [3]). Топология, порожденная такой метрикой, совпадает с введенной выше топологией прямого произведения. Положим $\rho_{\mathbf{a}}(x, x)=0, \rho_{\mathbf{a}}(x, y)=\max _{z \in X} a_{1}(z)$, если $C_{1}(x) \neq C_{1}(y)$, и $\rho_{\mathbf{a}}(x, y)=a_{k(x, y)}(x)$, где $k(x, y)=\max \left\{n: C_{n}(x)=C_{n}(y)\right\}$, в остальных случаях. Легко проверить, что $\rho_{\mathbf{a}}-$ метрика, согласованная с последовательностью а (см. [4]); при этом можно взять $b=1$. Если $a_{n}(x) \equiv \theta^{n}$, где $\theta \in(0,1)$, мы приходим к одной из «стандартных» метрик в пространстве $X$. Если $\lambda \in \mathscr{I}$ является $\tau$-инвариантной гиббсовской мерой с потенциалом конечной нормы, то последовательность функций $a_{n}(x):=\lambda\left(C_{n}(x)\right)$ обладает свойствами (а) $-(\mathrm{c})$ (соответствующую метрику $\rho_{\mathbf{a}}$ иногда называют метрикой Биллингсли, ср. $[6,7]$ ).

Множество метрик, согласованных с последовательностью а, обозначим через $\mathscr{R}(\mathbf{a})$.

Теорема 1. Пусть $f-$ регулярная функция, $\mu \in \mathscr{E}(f)$ u $\rho \in \mathscr{R}(\mathbf{a})$. Тогда $\operatorname{dim}_{\rho}(X(\mu))=h(\mu) / \gamma_{\mathbf{a}}$.

При доказательстве этой теоремы мы пользуемся тем, что $X(\mu) \subset X_{f, \mu(f)}$, и оцениваем $\operatorname{dim}_{\rho}\left(X_{f, \mu(f)}\right)$ и $\operatorname{dim}_{\rho}(X(\mu))$ сверху и снизу одной и той же константой $c=h(\mu) / \gamma_{\mathbf{a}}$, применяя в обоих случаях вариант теоремы 1.6 из [3], связывающей хаусдорфову размерность с локальной цилиндрической размерностью. Из двух упомянутых оценок более сложной является нижняя. Она основана на существовании такой меры $\nu \in \mathscr{P}$, что $\nu(X(\mu))=1$ и $\liminf _{n \rightarrow \infty} \frac{\ln \nu\left(C_{n}(x)\right)}{\ln a_{n}(x)} \geqslant c$. Если $\mu$ - эргодическая мера, ее можно взять в качестве $\nu$; в общем случае при построении меры $\nu$ (которая уже не будет $\tau$-инвариантной) мы используем идею Каяра [5], получившего результат, аналогичный теореме 1, в одномерном случае. Вначале фиксируем последовательность эргодических мер $\mu_{n}$, слабо сходящуюся 
к $\mu$ и обладающую двумя дополнительными свойствами. Первое из них состоит в том, что $\lim _{n \rightarrow \infty} h\left(\mu_{n}\right)=h(\mu)$. Второе касается характера зависимости друг от друга цилиндрических множеств, основания которых не пересекаются. Затем индуктивно строим достаточно редкую последовательность $\left\{T_{n_{i}}\right\}$ и определяем меру $\nu$ двумя условиями:

(i) если $C$ - цилиндр с носителем $T_{n_{i}} \backslash T_{n_{i-1}}\left(\right.$ где $\left.T_{n_{0}}=\varnothing\right)$, то $\nu(C)=\mu_{n_{i}}(C)$;

(ii) если $C^{\prime}, C^{\prime \prime}$ - цилиндры указанного вида, которым отвечают разные $i$, то они $\nu$-независимы.

Нетрудно убедиться, что при $d=1$ последовательность мер $\mu_{n}$ с требуемыми свойствами существует для любой меры $\mu \in \mathscr{I}$ (в качестве $\mu_{n}$ можно брать марковские меры растущего порядка). При $d>1$ мы можем лишь гарантировать, что такая последовательность существует для любой гиббсовской меры.

В ряде случаев теорема 1 позволяет вычислить размерность множества $X_{f, \alpha}$. Рассмотрим более общую задачу. Пусть функции $f_{i} \in \mathscr{C}(X), 1 \leqslant i \leqslant m$, удовлетворяют условию $(2)$ с одной и той же последовательностью $\left\{F_{n}\right\}$. Тогда для любого вектора $\boldsymbol{\beta}=\left(\beta_{1}, \ldots, \beta_{m}\right) \in \mathbb{R}^{m}$ функция $f_{\boldsymbol{\beta}}:=\sum_{i=1}^{m} \beta_{i} f_{i}$ удовлетворяет тому же условию.

Введем множество $E_{f_{1}, \ldots, f_{m}}=\left\{\left(\mu\left(f_{1}\right), \ldots, \mu\left(f_{m}\right)\right) \in \mathbb{R}^{m}: \mu \in \mathscr{E}\left(f_{\boldsymbol{\beta}}\right), \boldsymbol{\beta} \in \mathbb{R}^{m}\right\}$. Если $m=1$ и $f_{1}=f$, то, как нетрудно показать, $\operatorname{Int}\left(E_{f}\right)=(\underline{\alpha}, \bar{\alpha})$, где $[\underline{\alpha}, \bar{\alpha}]=$ $\{\mu(f): \mu \in \mathscr{I}\}$, причем концы этого отрезка допускают простые выражения в терминах давления:

$$
\underline{\alpha}=-\lim _{\beta \rightarrow+\infty} P(\beta f) / \beta, \quad \bar{\alpha}=-\lim _{\beta \rightarrow-\infty} P(\beta f) / \beta,
$$

а также в терминах эргодических средних:

$$
\underline{\alpha}=\liminf _{n \rightarrow \infty} \min _{x \in X} \frac{1}{\left|T_{n}\right|} \sum_{t \in T_{n}} f\left(\tau_{t} x\right), \quad \bar{\alpha}=\limsup _{n \rightarrow \infty} \max _{x \in X} \frac{1}{\left|T_{n}\right|} \sum_{t \in T_{n}} f\left(\tau_{t} x\right) .
$$

Приведенное описание множества $E_{f_{1}, \ldots, f_{m}}$ для случая $m=1$ легко обобщить на случай $m>1$.

Используя теорему 1 , можно также доказать следующее утверждение:

TеOPEMA 2. Если $\boldsymbol{\alpha}, \boldsymbol{\beta} \in \mathbb{R}^{m}, \boldsymbol{\alpha}=\left(\alpha_{1}, \ldots, \alpha_{m}\right), \boldsymbol{\beta}=\left(\beta_{1}, \ldots, \beta_{m}\right), \mu \in \mathscr{E}\left(f_{\boldsymbol{\beta}}\right) u$ $\mu\left(f_{i}\right)=\alpha_{i}, 1 \leqslant i \leqslant m, m o$

$$
\operatorname{dim}_{\rho}\left(\bigcap_{i=1}^{m} X_{f_{i}, \alpha_{i}}\right)=\frac{h(\mu)}{\gamma_{\mathbf{a}}}=\frac{1}{\gamma_{\mathbf{a}}}\left[\sum_{i=1}^{m} \beta_{i} \alpha_{i}+P\left(f_{\boldsymbol{\beta}}\right)\right]
$$

для любой метрики $\rho \in \mathscr{R}(\mathbf{a})$. Если $\boldsymbol{\alpha}$ не лежит в замыкании множества $E_{f_{1}, \ldots, f_{m}}$, mo $\bigcap_{i=1}^{m} X_{f_{i}, \alpha_{i}}=\varnothing$.

Для $d=1$ результаты, близкие к теореме 2, получены в [7-9] (при $m=1$ и различных условиях на $f$ ) и в [10] (при $m \geqslant 1$ ). Для $d \geqslant 1$ теорема 2 доказана в [11] при дополнительном предположении, что $\mu$ - эргодическая мера (это условие выполнено при любом $\boldsymbol{\beta}$, если $\boldsymbol{\beta} \mapsto P\left(f_{\boldsymbol{\beta}}\right)-$ гладкая функция).

\section{ЛИТЕРАТУРА}

1. Ruelle D. Thermodynamic Formalism. Addison-Wesley, Reading, MA, 1978. 2. Геopги Х.-О. Гиббсовские меры и фазовые переходы. Мир, М., 1992. 3. Темпельман А. A. Теор. вер. и прим., 44, вып. 3, 589-616 (1999). 4. Гуревич Б. М., Темпельман А. А. УМН, 54, вып. 2, 171-172 (1999). 5. Cajar H. Lect. Notes in Math., Vol. 892, Springer-Verlag, 
Berlin, 1988. 6. Olivier E. C. R. Acad. Sci. Paris, 326, Série I, 1171-1174 (1999). 7. Olivier E. C. R. Acad. Sci. Paris, 328, Série I, 13-16 (1998). 8. Takens F., Verbitski E. Comm. Math. Phys., 203, No. 3, 593-612 (1999). 9. Takens F., Verbitski E. On the variational principle for the topological entropy of certain non-compact sets. Preprint, 1999. 10. Fan Ai-Hua, Feng De-Jun, J. Statist. Phys., 99, No. 3/4, 813-856 (2000). 11. Tempelman A. A. J. Dynam. Control Systems, 7, No. 4, 535-551 (2001).

Московский государственный университет, механико-математический факультет e-mail: gurevich@mech.math.msu.su

Departments of Mathematics and Statistics, 7 августа 2001 г.

Penn State University, USA

e-mail: arkady@stat.psu.edu

УДК $517.927+517.928$

\title{
О модельной задаче для уравнения Орра-Зоммерфельда с линейным профилем*
}

\author{
(c) 2002. А. В. Дьяченко, А. А. ШкАликов
}

В заметке рассматривается спектральная задача

$$
\begin{aligned}
& -i \varepsilon y^{\prime \prime}=(x-\lambda) y, \\
& y(-1)=y(1)=0,
\end{aligned}
$$

где $\lambda-$ спектральный параметр, а $\varepsilon>0-$ малый параметр. Эта задача является моделью для более сложной задачи Орра-Зоммерфельда с линейным профилем (течение Куетта).

Заметим, что задача Орра-Зоммерфельда после элементарных преобразований приводится к виду

$$
\begin{gathered}
-i \varepsilon\left(z^{\prime \prime}-\alpha^{2} z\right)=(x-\lambda) z \\
\int_{-1}^{1} z(t) \operatorname{sh} \alpha(1-t) d t=0, \quad \int_{-1}^{1} z(t) \operatorname{sh} \alpha(1+t) d t=0 .
\end{gathered}
$$

Здесь $\varepsilon=1 / \alpha R, R$ - число Рейнольдса, а $\alpha-$ волновое число. Важным является изучение поведения спектра задачи (3), (4) при $R \rightarrow \infty$ (см., например, [1] и [2]). Здесь мы проведем исследование поведения спектра при $\varepsilon \rightarrow 0$ только для модельной задачи (1), (2). Характер поведения спектра задачи (3), (4) при $\varepsilon \rightarrow 0$ в главном сохраняется, хотя возникают новые интересные явления. Подробное изложение результатов по задаче Орра-Зоммерфельда будет проведено в нашей следующей работе.

В работе [3] было доказано, что собственные значения задачи (1), (2) при $\varepsilon \rightarrow 0$ локализуются вдоль луча $[-i / \sqrt{3},-i \infty)$ и двух отрезков $[1,-i / \sqrt{3}]$ и $[-1,-i / \sqrt{3}]$ (см. рис. 1). В [3] была найдена также асимптотика собственных значений в окрестности указанных отрезков. Цель этой заметки - вычислить асимптотику собственных значений задачи $(1),(2)$ по малому параметру $\varepsilon$ на луче

* Работа выполнена при поддержке грантов РФФИ № 01-01-00691 и 00-15-96100. 